\title{
The effect of rotation on the spectrum of $\mathrm{Vega}^{8}$
}

\author{
Jinmi Yoon ${ }^{1,2}$, Deane M. Peterson ${ }^{1,3}$, Robert J. Zagarello ${ }^{4}$, J. Thomas Armstrong ${ }^{5,6}$, and \\ Thomas Pauls ${ }^{5,7}$
}

\begin{abstract}
The discovery that Vega is a rapidly rotating pole-on star has raised a number of questions about this fundamental standard, including such issues as its composition, and in turn its mass and age. We report here a reanalysis of Vega's composition. A full spectral synthesis based on the Roche model derived earlier from NPOI interferometry is used. We find the line shapes in Vega's spectrum to be more complex than just flat-bottomed, which have been previously reported; profiles range from slightly self-reversed to simple "V" shapes. A high SNR spectrum, obtained by stacking spectra from the ELODIE archive, shows excellent agreement with the calculations, provided we add about $10 \mathrm{~km} \mathrm{~s}^{-1}$ of macroturbulence to the predicted spectra. From the abundance analysis, we find that Vega shows the peculiar abundance pattern of a $\lambda$ Bootis star as previously suggested. We investigate the effects of rotation on the deduced abundances and show that the dominant ionization states are only slightly affected compared to analyses using non-rotating models. We argue that the rapid rotation requires the star be fully mixed. The composition leads to masses and particularly ages that are quite different compared to what are usually assumed.
\end{abstract}

Subject headings: line: profiles — stars: abundances — stars: chemically peculiar — stars: early-type — stars: individual (Vega) — stars: rotation

\section{Introduction}

With the announcement of the detection of the interferometric signature of rapid rotation in Vega (Peterson et al. 2004, 2006b; Aufdenberg et al. 2006), a number of questions were raised about the fundamental standard. Earlier suggestions of rapid rotation were based on the high luminosity Petrie 1964; Grav 1988) of the ob-

\footnotetext{
${ }^{1}$ Department of Physics and Astronomy, Stony Brook University, Stony Brook, NY 11794-3800

2 jyoon@grad.physics.sunysb.edu

${ }^{3}$ dpeterson@astro.sunysb.edu

${ }^{4}$ PCPION, South Setauket, NY 11720-1325; rzagarello@mail.astro.sunysb.edu

${ }^{5}$ Remote Sensing Division, Code 7215, Naval Research Laboratory, 4555 Overlook Avenue SW, Washington, DC 20375

${ }^{6}$ tom.armstrong@nrl.navy.mil

${ }^{7}$ pauls@nrl.navy.mil

${ }^{8}$ Based on spectral data retrieved from the ELODIE archive at Observatoire de Haute-Provence (OHP)
}

ject and the unusual shapes of the weak lines in the spectrum (Gulliver. Hill. \& Adelman 1994; Hill. Gulliver, \& Adelman 2004). The high luminosity is immediately explained using Roche models for the figure of the rotating star, von Zeipel's theorem (von Zeipel 1924) to characterize the temperature distribution, and adopting a nearly pole-on geometry, required by the small line widths (Grav 1988).

But this model, a star rotating near breakup, raises the question of whether such fundamental issues as Vega's composition, mass, and age, are accurately known. It has been recognized for some time that Vega appears metal poor (Sadakane \& Nishimura 1981; Adelman \& Gulliver 1990). And although it has been known since the early 20th century that masses deduced from luminosity and radius measurements are strongly affected by composition, recent mass and age determinations have largely assumed solar composition, the assumption being that sharp lined A 
stars often show abundance peculiarities that are assumed due to diffusion and generally confined to surface layers. The recognition of rotation velocities approaching breakup renders that assumption unlikely, since rotation driven circulation is likely to mix the envelope completely and deeply over times short compared to operable diffusion timescales.

Furthermore, the large surface temperature gradients that would be associated with high rotation raise a new question: how seriously are simple, single model atmosphere analyses of the spectrum affected by the composite nature of the atmosphere? The peculiar line shapes add to this concern. A full analysis of the spectrum, or at least representative spectral features, seems necessary to demonstrate that we understand the peculiar line profiles and are able to derive reliable abundances.

This in turn requires high resolution, low Hill, Gulliver, \& Adelman (2004). The spectra of Vega available on the ELODIE archive provide us the necessary resolution and low noise, we describe those data in $\S 2$. The computation of the synthetic spectra based on a rotational model are described in $\S 3$ and the deduced abundances and other characteristics we reported in $\S 4$, including the discovery that significant macroturbulence must be adopted. In $\S 5$ we discuss the implications of the abundance profile and argue that the suggestion that Vega belongs to the $\lambda$ Bootis class of objects is probably correct. We note that the effect of rotation on the line strengths depends strongly on the line considered and propose a simple resolution to the prediction of large departures from LTE in the Fe I spectrum that have not been seen in practice. We examine more closely the issue of rotational mixing and conclude that the abundances we find here likely represent the material out of which Vega was formed. Lastly, we estimate the mass and age of Vega based on this composition.

After submitting this manuscript we became aware of a paper Takeda. Kawanomoto. \& Ohishi 2008) that had been recently accepted for publication in this Journal which undertakes an analysis noise; spectra comparable to those used by of their previously published (Takeda, Kawanomoto.

1994; Hill. Gulliver. \& Adelman 2004). These authors draw a number of conclusions in agreement with what we find here. But they also arrive at quite a different physical model of Vega, concluding in the process that errors were made in the reductions of the published interferometry. We will comment on these results at the appropriate points.

\section{The Observational Data}

The Vega spectra we used are from the ELODIE archive (Moultaka et al. 2004), which contains high-resolution $(R \sim 42,000)$ echelle spectra from the ELODIE spectrograph obtained at the Observatoire de Haute-Provence $1.93 \mathrm{~m}$ telescope. The ELODIE data pipeline automatically extracts the spectra, establishes the dispersion, and corrects for scattered light. The spectra used here were obtained between 1996 and 2004. The wavelength rectified spectra covering $\lambda \lambda 4000-6800$ are provided with $0.05 \AA$ sampling.

Barycenter corrections were required before coadding the spectra. To improve the signal-to-noise ratio (SNR) which is 250 for a typical spectrum, we co-added 49 out of 71 available spectra. In the process we rejected spectra whose SNR was less than 100 and those showing noticeable fringing. We also replaced bad pixels whose residuals in individual spectra were 5 times larger than the typical noise by interpolating adjacent pixels. The co-added spectra were converted to a residual intensity scale by normalizing them to the scale of the synthetic spectra described in $\S 3$. The resultant spectra, segments of which are shown in Figures 11 and 2, were then compared to the synthesized spectra for the abundance analysis. The SNRs of the co-added spectra were estimated to range from 750 to 2,200 depending on the spectral regions.

Besides the ELODIE spectra, spectra of comparably high SNR and resolution of Vega have been obtained at the Dominion Astrophysical Observatory (DAO; Gulliver. Hill. \& Adelman 1994; Hill. Gulliver, \& Adelman 2004) and the Okayama Astrophysical Observatory (OAO; Takeda, Kawanomoto, \& Ohishi 2007). The DAO spectra (SNR 3,300) have not hishil been released publicly. However they are avail-

2007) spectra toward understanding Vega's rota-

tion, much along the lines taken earlier (Gulliver, Hill, \& Adelman 

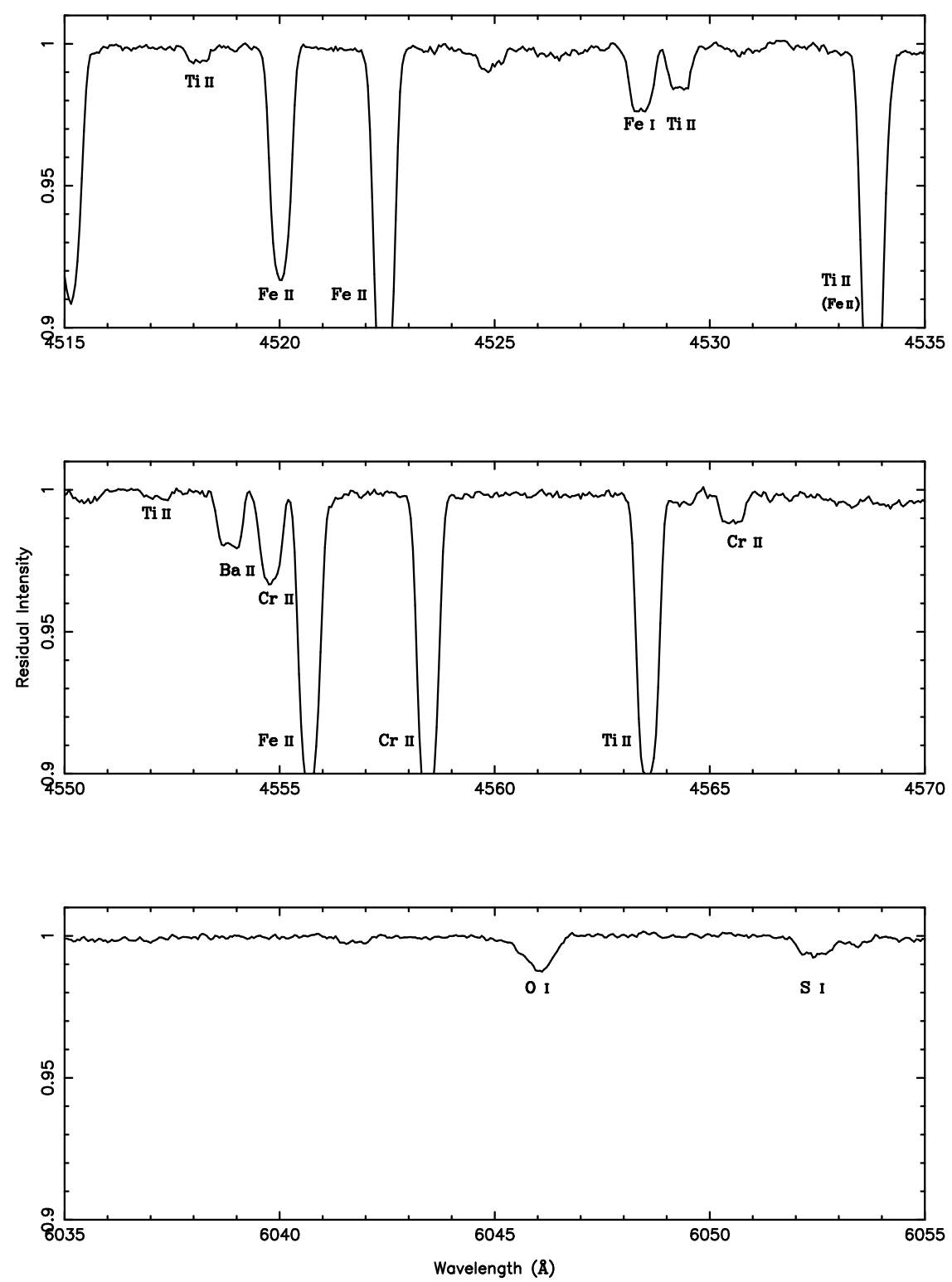

Fig. 1.- Shown are line profiles representative of the range of shapes encountered for weak lines in the ELODIE spectra of Vega. The shapes run from weakly "self-reversed" (e.g., Fe I $\lambda 4528$ and Ba II $\lambda 4554$ ) through flat-bottomed (Cr II $\lambda 4565$ and S i $\lambda$ 6052) to "V"-shaped (O I $\lambda$ 6046). Where known, blends are indicated in parenthesis. Wavelengths are in the star's rest frame. 

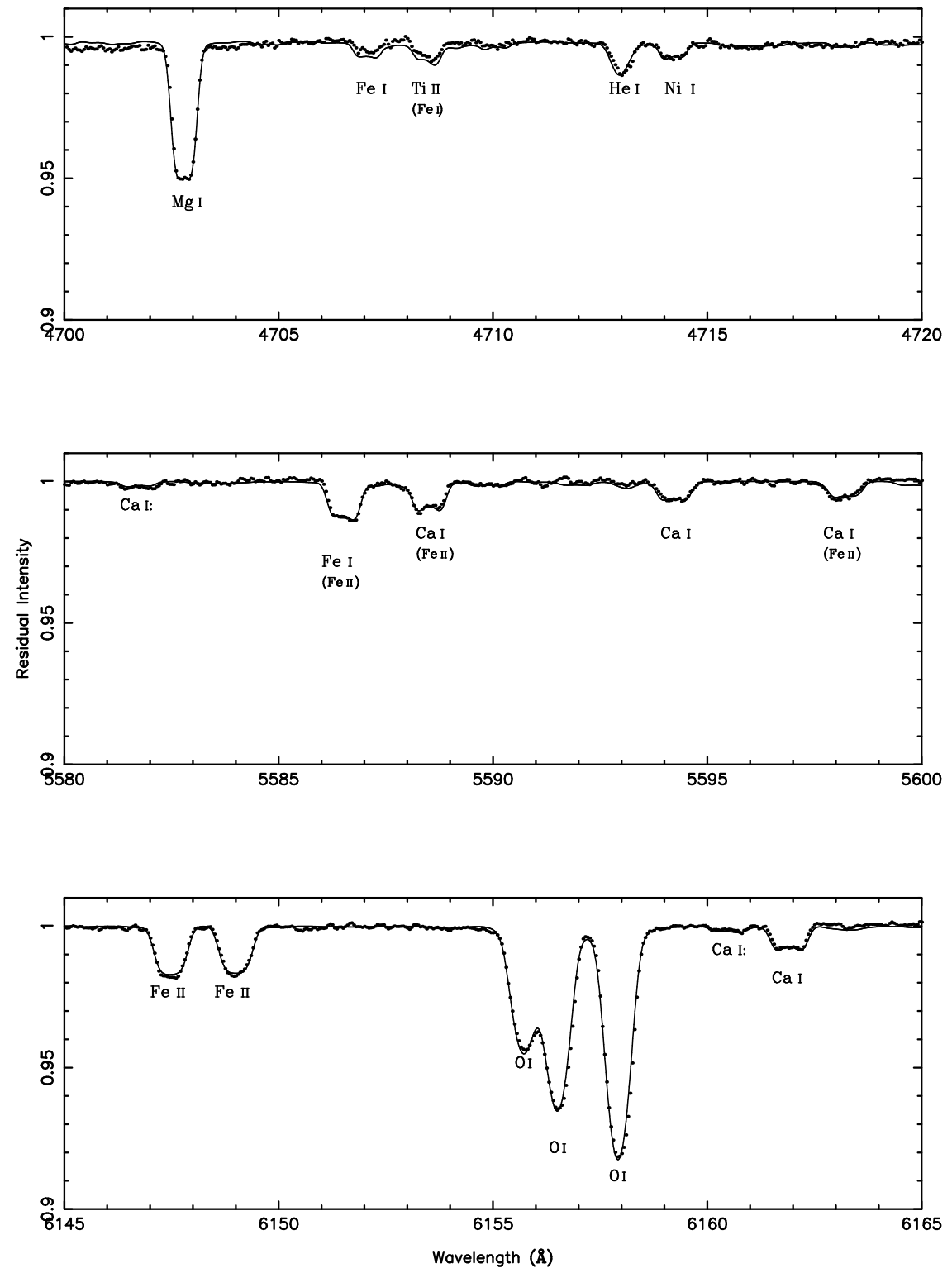

Fig. 2.- Plotted here are additional segments of spectra (dotted lines) showing the range of shapes of weak lines, as in Figure1, only now overplotted with the synthetic spectra (continuous lines). Note particularly He I $\lambda 4713$ which, with an excitation potential of $21 \mathrm{eV}$, is formed in a small region around the rotational pole and displays the corresponding "V" shape. At the other extreme Ca I $\lambda 6162$ shows the weak double-horned ("self-reversed") shape reflecting its very low excitation potential, $1.9 \mathrm{eV}$; it is contributed exclusively by the cooler equatorial regions. Other lines showing this behavior are Ti II $\lambda 4708$, Fe I $\lambda 5586$, and $\mathrm{Ca}$ I $\lambda 5588$, although all three are (weakly) blended. Two iron lines, Fe II $\lambda \lambda 6147$ and 6149 , at intermediate excitations of $3.9 \mathrm{eV}$ above the $7.8 \mathrm{eV}$ ionization potential of Fe $\mathrm{I}$, show the expected flat-bottomed shapes, although seen against a slight variability in the background continuum. The weak Ca I lines indicated with ":" were not included in the abundance determination. 
able as part of a graphic toolkit 11 which allows one to examine sections of spectra at high resolution and identify lines and probable blends. We made extensive use of this tool during this investigation $\sqrt[2]{2}$

Also recently published are OAO spectra (SNR from 1,000 to 2,000 on average) covering $\lambda \lambda 3900$ 8800. However, these spectra display emission (e. g., $\sim \lambda 4560$ ) and absorption (e. g., $\sim \lambda 6060$ ) features and show the head of the Paschen continuum to be strongly in emission, features not reported elsewhere. So we have chosen to focus exclusively on the ELODIE data set.

\section{Computations}

We assume Vega can be described by a gravitydarkened Roche spheroid in solid-body rotation, with a point mass gravitational potential, showing a temperature distribution varying according to von Zeipel's theorem (von Zeipel 1924), and seen nearly pole-on (e. g., Peterson et al. 2006b; Aufdenberg et al. 2006). Because the recent interferometric measurements taken at the Navy Prototype Optical Interferometer (NPOI; Armstrong et al. 1998) and the Center for High Angular Resolution Astronomy (ten Brummelaar et al. 2005) array yield closely similar model parameters, we adopt the parameters obtained from the NPOI data (Peterson et al. 2006b) for synthesizing spectra; the model has a fractional rotation velocity, $\omega=0.926$, a polar surface gravity of $\log g_{\mathrm{p}}=4.074$, a polar effective temperature of $T_{\mathrm{p}}=9988 \mathrm{~K}$, an inclination of the rotational axis to the line of sight $i=4.54^{\circ}$, and a projected rotational velocity of $v \sin i=21.7 \mathrm{~km} \mathrm{~s}^{-1}$. For details of fitting Roche models to the NPOI data see Peterson et al. (2006a), and for issues specific to Vega see Peterson et al. (2006b), respectively.

To calculate the emergent spectrum we constructed a square $256 \times 256$ grid which contains the apparent disk of the star, calculated the stellar parameters at the center of each cell that actually fell on the flattened disk, and computed an emergent

${ }^{1}$ http://www.brandonu.ca/physics/gulliver/ccd_atlases.html

${ }^{2}$ At the same time R.L. Kurucz (2007, private communication) provided a high resolution synthesized spectrum for Vega in the $450-500 \mathrm{~nm}$ region based on a line list and gf values calibrated to a solar spectrum which proved extremely useful. flux as a function of $\lambda, \mu$ (cosine of the angle between the local normal and the line of sight), $T_{\text {eff }}$, $g_{\text {eff }}$ (local gravity reduced by centrifugal force), and projected velocity using the ATLAS9 model atmosphere grid (Castelli \& Kurucz 2003) and the atomic line data given in the extensive compilation of Kurucz \& Bell (1995). The fluxes were integrated over the disk to yield the synthetic model spectrum. In these calculations LTE, hydrostatic equilibrium, and plane-parallel atmospheres were assumed to represent the star's surface locally.

A concerns have been raised recently (Aufdenberg et al. 2006; Monnier et al. 2007) about the rigorous applicability of the von Zeipel theorem in the parts of the disk of a rotating star that are rendered cool enough to generate convection. We believe the issue is not relevant to Vega. In our model the temperature drops to about $7600 \mathrm{~K}$ at the equator, and the effective gravity in turn decreases to about $\log g \sim 3.5$. From a model atmosphere with $T_{\text {eff }}=7500 \mathrm{~K}, \log g=3.5$ we find the reduced density and in turn increased fraction of hydrogen ionized compared to the main sequence, substantially decreases the extent of the convective region and the efficiency of the resulting convection. Convection carries significant flux only in the range of $1 \leq \tau_{\text {Rosseland }} \leq 30$, well out from the interior where the flux requirement is established.

\section{Results}

\subsection{Line Shapes}

The abundance analysis was done by adjusting each element abundance until the model spectra fit the co-added spectra. Since Vega's lines are sharp and blending is minimal, the process of adjusting the abundances was straightforward. Several representative regions of the coadded ELODIE spectrum are shown in Figures 11 and 2. Weak lines throughout the spectrum show not only the flat-bottomed shapes (Cr II $\lambda 4565$, S I $\lambda 6052$, and Fe II $\lambda 6147)$ as noted in recent studies (Gulliver. Hill. \& Adelman 1994; Hill. Gulliver, \& Adelman 2004) but also weakly "self-reversed" shapes such as Mg I $\lambda 4702$ and Ca I $\lambda 6162$ and "V" shapes such as He I $\lambda 4713$ and O I $\lambda 6046$.

The unusual shapes of the weak lines are strongly correlated with excitation and ionization potential and can be understood in terms of 
how the Boltzmann factors amplify the temperature gradient across the disk. Since Vega is seen nearly pole on, the center of the apparent disk is almost exactly at one pole, the hottest point on the star. On the other hand, the limb is nearly the equator which is not only $2,400 \mathrm{~K}$ cooler than the pole, but the visible gas is actually cooler still owing to the simple projection effects associated with limb-darkening. Therefore the bound states responsible for the lines seen from the light elements such as He I, O I, Mg II, Al II, and Si II whose ionization and excitation potentials are quite high are excited mostly at the axis with zero projected velocity. There is almost no contribution to the line profiles from the rotationally shifted equatorial region, resulting in "V" shapes. The lower the excitation potentials the lines have, the more enhanced the contribution from the more rapidly rotating equatorial regions becomes and the wider and more square shaped the line profiles get. For the elements such as Ca I, Fe I, and Ba II with the lowest excitation potentials one sees a mild doublehorned shape ("self-reversed") as the contribution from the equatorial region completely dominates the profile. In this sequence the flat-bottomed shape is formed at intermediate excitation potentials such as those of the lines of $\mathrm{Cr}$ II and Fe II. Our synthetic spectra predict well this sequence of line shapes as shown in Figure 2, where three regions of the ELODIE spectrum, overplotted with our synthetic spectrum, are shown. We see that weak Fe II lines tend to have a flatbottomed shape while weak Fe I lines show a selfreversed shape. Examination of data presented by Gulliver. Hill, \& Adelman (1994) and particularly in Hill, Gulliver, \& Adelman (2004) suggests these shapes are present in their data as well.

\subsection{Macroturbulence}

In the process of the spectral synthesis, in order to fit the shapes of the weak line we found we had to reduce the resolution of the spectra well below the nominal resolution of 42,000 of the ELODIE spectra, ultimately adopting a resolution of about 25,000 as shown in Figure 3. We interpret this additional broadening, which was accomplished by convolving the synthetic spectrum with a Gaussian, as adding $10 \mathrm{~km} \mathrm{~s}^{-1}$ of macroturbulence to the nominal ELODIE resolution (also assumed to be a Gaussian). The effect of this addi- tional broadening is most noticeable in steep-sided line profiles (e. g., $\mathrm{Mg}$ I $\lambda 4703$ and $\mathrm{Ni}$ I $\lambda$ 4713), as shown in Figure 3 . As a result the value determined for the macroturbulence comes from low excitation lines and hence refers more to the equatorial regions than the polar regions. As might be expected, "V" shaped lines such as He I $\lambda 4713$ are insensitive to the macroturbulence, as also shown in Figure 3. This is a very interesting result which we discuss at more length below. One caution is immediately apparent though: line widths might not be reliable indicators of actual projected velocity, at least for stars seen at low inclination.

At this point our analysis deviates sharply from the recent contribution from Takeda. Kawanomoto, \& Ohishi (2008), who seem not to have considered the possibility of large scale non-thermal line broadening. That there could, and even should, be turbulence on large scales in the atmosphere of Vega seems easy to justify. Even very slow, $\mathrm{cm} \mathrm{s}^{-1}$, subsurface circulation currents will be magnified by the many order of magnitude drop in density found in the outer envelope, as required by the equation of continuity. Add to this a very strong Coriolis force owing to the rapid rotation and a surface covered with large eddies cyclones - is to be expected. Ignoring this possibility, Takeda, Kawanomoto. \& Ohishi (2008) were forced to adopt a relatively slowly rotating model, creating a clear conflict with the interferometric measurements (Peterson et al. 2006b; Aufdenberg et al. 2006).

\subsection{Abundance Analysis and Microturbu- lence}

As is often the case, we found that it was generally not possible to find abundances for elements (or even the same ion of an element) which gave good fits to both strong and weak lines simultaneously. This is usually taken as a signal that some microturbulence needs to be introduced. To this end, we determined the abundances from the Fe II lines for two choices of the microturbulence, as shown in Figure 4. Here the abundances are given as the logarithm of the ratio of the number of an element to that of total elements, $\log \frac{N_{\text {el }}}{N_{\text {e }}}$. Castelli \& Faraggiana (1979) and Sadakane \& Nishimura (1981) have previously noted that the influence of the microturbulence is less important in the visual region for 

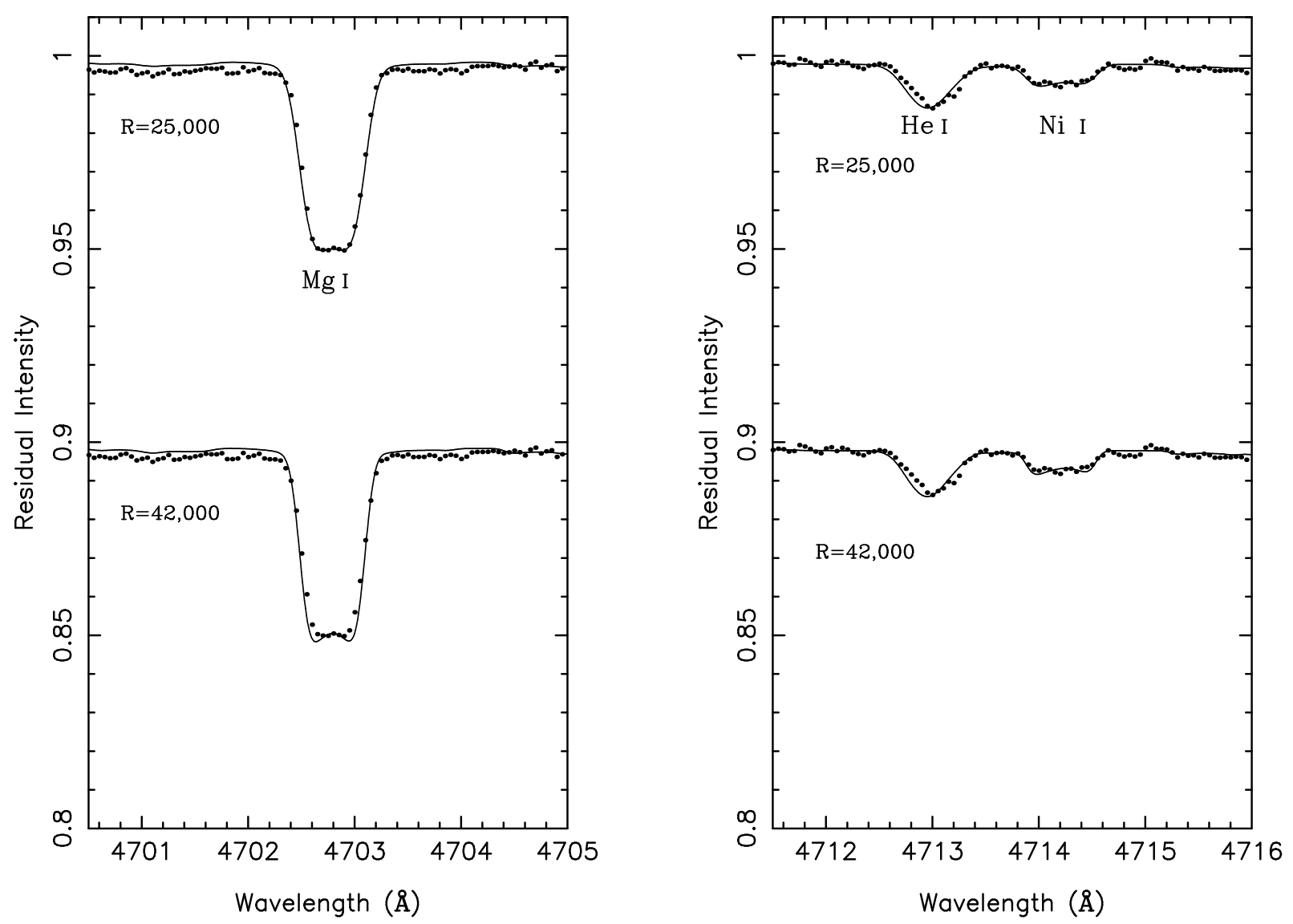

Fig. 3.- These figures plotted as in Figure 2 illustrate the need for line broadening in addition to rotation and microturbulence (in both panels the lower spectra are offset by 0.1). Mg I $\lambda 4702$ shows the problem most clearly although it is also evident in Ni I $\lambda$ 4714. The nominal ELODIE resolution of 42,000 (assumed to be Gaussian) allows too much structure in the steep-sided line profiles. Reducing the resolution to 25,000 appears to be required, which we interpret as a contribution of about $10 \mathrm{~km} \mathrm{~s}^{-1}$ of macroturbulence. The effect of adding this macroturbulence is to improve the fit dramatically in the bottoms of the weak, lowexcitation lines while causing the line widths to be a bit wide. This suggests the actual projected rotation rate is below the adopted $21.7 \mathrm{~km} \mathrm{~s}^{-1}$, as was suggested in the initial interferometric data reductions reported by Peterson et al. (2006b). Note that high excitation lines like He I $\lambda 4713$ are not affected by the added macroturbulence. 
lines of intermediate strengths about $40 \sim 70 \mathrm{~m} \AA$, which we also found. For Fe II, which has the widest range of equivalent widths, we find both the scatter and any trend with equivalent width are significantly reduced for a microturbulence of about $2 \mathrm{~km} \mathrm{~s}^{-1}$ which we subsequently adopt. The $\mathrm{O}$ I triplets also support $2 \mathrm{~km} \mathrm{~s}^{-1}$ (e. g., Figure 2 which shows only the case for $\left.2 \mathrm{~km} \mathrm{~s}^{-1}\right)$.

Table 1 shows the deduced abundances for Vega with a microturbulence of $\xi_{T}=2 \mathrm{~km} \mathrm{~s}^{-1}$. In selecting lines we eliminated severe blends but included weak blends where we felt reliable abundances could be obtained. The columns are the laboratory wavelength, lower excitation potential, equivalent width, $\log g f$, and the deduced abundance $\left(\log \frac{N_{\text {el }}}{N_{\text {tot }}}\right)$. Blends we have decided to retain are noted in the last column. The abundances for elements with only single lines such as $\mathrm{Al}$ II, S I, $\mathrm{Mn}$ I, and Ni I must be considered uncertain. Even where there was no obvious blending, abundances were determined exclusively by spectral synthesis. Nevertheless, we give equivalent widths for comparison with recent work; agreement is within $1-2 \mathrm{~m} \AA$ typically. Equivalent widths are missing where lines were not able to be measured due to "one-sided" blends or difficulty in defining the local continuum level.

Notable in Table 1 is the discrepancy between Fe I and Fe II abundances. The abundances of Fe reported by Adelman \& Gulliver (1990) do not show this dramatic lack of balance, and this might be viewed as supporting the smaller temperature gradient derived by Gulliver. Hill, \& Adelman (1994) and Hill. Gulliver. \& Adelman (2004). In contrast with $\mathrm{Fe}$, the abundances of $\mathrm{Mg}$ I and $\mathrm{Mg}$ II shown in Table 1 do not show similar behavior. We discuss this result further below.

\section{Discussion}

\subsection{How Does Rotation Affect Abun- dances?}

The main difference between a pole-on rapidly rotating star, as modeled here, and a classical plane-parallel stellar atmosphere model is that for the same integrated colors, the rotating model has some fraction of its surface at both higher and lower local effective temperatures than the nonrotating model. The expected effect is that there will be spectral lines in a range of excitation and
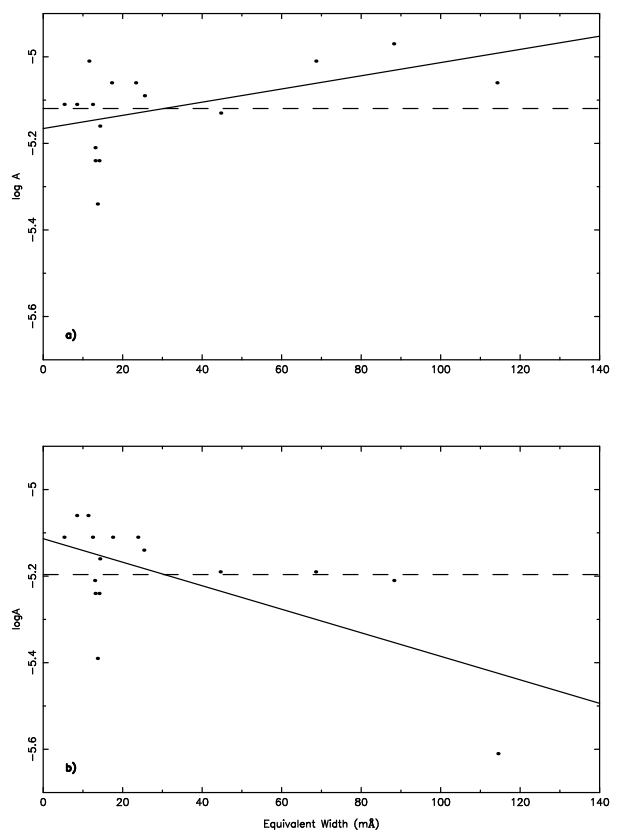

Fig. 4.- These plots show the derived abundances (data points) versus equivalent widths of Fe II lines for two different assumed values of the microturbulence. Panels a) and b) show the derived abundances for microturbulence values of $2 \mathrm{~km} \mathrm{~s}^{-1}$ and $4 \mathrm{~km} \mathrm{~s}^{-1}$, respectively. The dashed line shows the unweighted average abundances and the solid line shows the trend with equivalent width. We adopt a microturbulence of $2 \mathrm{~km} \mathrm{~s}^{-1}$ in our abundance determinations. 
ionization energies where the two models give similar results. For Vega this is the case when the sum of the excitation energy and ionization energy (for lines of ions such as Fe II) is about $10 \mathrm{eV}$. But for both higher and lower energy features the expanded range of temperatures will enhance line strengths, resulting in a decrease in the deduced abundances from those lines (described as "intensification" by Takeda, Kawanomoto, \& Ohishi 2008).

We see that trend here. Table 2 summarizes the results of two recent analyses of Vega with non-rotating models (Sadakane \& Nishimura 1981; Adelman \& Gulliver 1990) along with the element-by-element results determined here. Our results from $\mathrm{Mg}$ I, $\mathrm{Mg}$ II, $\mathrm{Al}$ II, Si II, Ti II, Cr II, Mn I, and Fe II are about -0.6 dex below solar, roughly that found by earlier authors. These are mostly the dominant ionization stages and thus yield fairly stable abundances. Other ions, including $\mathrm{C}$ I, Ca I, Sc II, Fe I, Ni I, and Ba II are deficient by about -1.0 dex, or even more, than the solar abundances ( $\mathrm{C}$ I by -0.6), and are depressed by typically several tenths dex compared to the earlier studies, in this case because of their relatively small excitation and ionization energies.

On the other side, the He abundance we deduce, $N_{\mathrm{He}} / N_{\text {tot }}=0.072 \pm 0.004$, which is essentially solar (0.078), is substantially higher than that found by Adelman \& Gulliver (1990), running counter to expectations. The result determined here is based on five of the six lines in the ELODIE spectral window that are expected to be measurable ( $\lambda 5875$ is heavily involved with atmospheric water vapor lines), while the helium abundance of Adelman \& Gulliver (1990) is based on $\lambda 4471$ only. Otherwise, we have no explanation for why we obtain a larger helium abundance.

In broadest terms, we find that if one can determine abundances from lines of the dominant ionization stage of an element, the errors induced by not accounting for rotation are small. Where lines from the dominant ionization stage are not accessible (e. g.,Ba III), one can expect large corrections to be required when standard, modelatmosphere analyses are applied to objects rotating near breakup.

One interesting example of the problems that can arise because of the corrections required between different ionization stages of the same ele- ment, involves the ionization balance between Fe I and Fe II. Problems with the Fe I/Fe II ionization balance have been reported for a wide range of stars (e. g., Gigas 1986; Allende Prieto et al. 1999; Thévenin \& Idiart 1999; Johnson 2002). For Vega, departures from LTE are predicted to produce about 0.3 dex errors in abundances deduced from Fe I lines while Fe II lines are barely affected Gigas 1986). However, these calculations are difficult owing to the complexity of the atom and the lack of accurate collision and photoionization cross sections. For example, Pradhan et al. (1995) have found that many of the photoionization cross sections of Fe I are significantly higher than those previously adopted (e. g., Gigas 1986) with the possibility that the actual corrections from departures from LTE are larger still.

The problem with the Fe balance in Vega is confusing since at first glance, straightforward LTE analyses (Sadakane \& Nishimura 1981; Adelman \& Gulliver 1990) provide apparent agreement between the abundances deduced from the two ions. This is in contrast to the sizable departures from LTE required in other similar objects.

However, even though we assume LTE in our analysis here, we also find a serious iron ionization imbalance amounting to $\sim 0.4 \mathrm{dex}$, but in the opposite sense of that induced by non-LTE. To understand the origin of this imbalance we reanalyzed representative lines from the two iron ionization states and, as a check, from the two magnesium ions present, using a standard plane-parallel model. We find that rotation induces an apparent 0.35 dex error in the Fe I/Fe II ionization balance, while the corresponding effect in the $\mathrm{Mg}$ I/Mg II balance is only about 0.1 dex.

Thus we reach the amusing conclusion that a simple LTE analysis of Vega using models which do not account for rotation give a good ionization balance because of a nearly complete cancellation of the effects of photoionization-driven departures from LTE in the Fe I ion, on the one hand, and an enhanced Fe I line spectrum contributed by the extensive cool equatorial regions of the model owing to the favorable viewing geometry, on the other. Note however, the near balance between these two effects may disappear when one analyses lines in either the ultraviolet or infrared, owing to the changing relative contribution of the equatorial regions to the overall light. 
We note that Takeda. Kawanomoto. \& Ohishi (2008) have independently commented on the near cancellation of departures from LTE versus the effects of rotation in the iron ionization balance. However, in their calculation the rotation induced errors are predicted to be about half those calculated here, owing to the much lower rotation velocity and the corresponding dramatically reduced temperature gradient $(\sim 900 \mathrm{~K})$ in their model.

\subsection{Is Vega a $\lambda$ Bootis Star?}

Since Baschek \& Slettebak (1988) remarked that Vega showed an abundance pattern similar to the $\lambda$ Bootis stars, several studies (e. g., Venn \& Lambert 1990; Iliiić et al. 1998) reported that Vega may be a mild $\lambda$ Bootis star. We confirm that result here. The abundance pattern we deduce matches well the main characteristics of the abundance patterns of $\lambda$ Bootis stars as summarized, for example by Heiter (2002). Elements such as $\mathrm{Si}, \mathrm{S}, \mathrm{Ca}$, and $\mathrm{Sc}$ fall in the middle of their respective typical ranges while $\mathrm{O}, \mathrm{Mg}, \mathrm{Ti}$, $\mathrm{Cr}, \mathrm{Mn}$, and $\mathrm{Fe}$ are on the high side of normal and $\mathrm{Ni}$ and $\mathrm{Ba}$ are on the low side. While most elements fit the $\lambda$ Bootis abundance pattern well, $\mathrm{C}$ and $\mathrm{Al}$ are somewhat out of the reported range. The $\mathrm{Al}$ abundance is based on one line and is not certain, while carbon is off the lower end of the pattern reported by Heiter (2002). However Pauzen et al. (1999), in an extensive discussion of carbon and oxygen in this group of objects, find several objects with carbon abundances as low as -0.7 dex with respect to the Sun. We conclude that Vega would not be rejected as a $\lambda$ Bootis star on the basis of its carbon abundance and the rest of the abundances determined here are very much in keeping with membership in this group.

\subsection{Is Vega Well Mixed?}

A presumption, often unstated, about the nature of $\lambda$ Boo stars is that the deviations from solar composition are limited to surface layers (e. g., Baschek 1992; Holweger \& Stürenburg 1993), much the same as has been concluded for the Ap and Am stars which also occupy this part of the $\mathrm{H}$ $\mathrm{R}$ diagram. But there has always been some concern about that assumption since unlike the latter groups the $\lambda$ Boo stars appear to have a distribution of rotation velocities similar to normal stars (e. g., Holweger \& Stürenburg 1993).
We argue here that since Vega is rotating at a significant fraction of breakup and yet displays fairly typical $\lambda$ Boo characteristics, it is unlikely that these composition anomalies are limited to the surface; more likely, Vega is well mixed. The literature on rotationally induced mixing has generally focused on the surface layers and the question of whether the Ap and Am phenomena could be understood as due to diffusive separation (e. g., Charbonneau 1993) and not on how fast an inhomogeneity introduced on the surface would be mixed throughout the envelope.

However, recent efforts to include the effects of rotation in evolutionary calculations of massive stars (Meynet \& Maeder 1997) have led to an examination of how inhomogeneities will be redistributed through a star (Talon et al. 1997; Ekström et al. 2008), suggesting that extensive mixing is to be expected. In fact, at the highest velocities in models down to $3 M_{\odot}$, the lowest mass examined, the mixing is predicted to be so deep there is the possibility that some of the nuclear products from the $\mathrm{CNO}$ burning region might be mixed to the surface.

This is an interesting possibility, given the low carbon abundance we have found. From this point of view, missing is an estimate of the nitrogen abundance, the lines of which are out of the ELODIE spectral range. However measurements of nitrogen line equivalent widths have been reported elsewhere. To fill in the abundance of this important nuclide taking full account of the effects of rotation, we have calculated the abundances for the nitrogen equivalent widths reported in Venn \& Lambert (1990) for $\lambda 7442.28$ $\left(\log N / N_{\text {tot }}=-4.05,\left[N / N_{\text {tot }}\right]=+0.07\right)$ and $\lambda 7468.29\left(\log N / N_{\text {tot }}=-4.02,\left[N / N_{\text {tot }}\right]=+0.1\right)$ ( $\lambda 7423$ appears to be blended and we exclude it here), finding values quite close to those deduced by Venn \& Lambert (1990) at about 0.085 dex above solar. This is an intriguing result. Although it is difficult to know what "normal" is in this star, normalizing to oxygen gives $[\mathrm{N} / \mathrm{O}] \sim+0.2$ and $[\mathrm{C} / \mathrm{O}] \sim-0.5$, which may very well indicate that some $\mathrm{CN}$ cycle processed material has been mixed into the envelope of the star. In this regard we note that Vega represents a rather unique object; a few other $\lambda$ Boo objects have projected velocities in the vicinity of $200 \mathrm{~km} \mathrm{~s}^{-1}$, but Vega is the one object known to rotate as fast as $275 \mathrm{~km} \mathrm{~s}^{-1}$, 
less than $10 \%$ from breakup in terms of angular velocity. However, without a better understanding of the composition of the material Vega started with, or other supporting information, we must leave this as just an intriguing possibility.

In summary, we believe a fairly strong case can be made for the outer layers of Vega being well mixed, possibly even down to the edge of its nuclear burning core. If this is so then we are looking at about $2 M_{\odot}$ of material of highly unusual composition in an object that is much too young to display such extreme depletion in heavy elements. In this case the various mechanisms put forth to explain the $\lambda$ Boo phenomena that rely on its being limited to the superficial layers (e. g., Kamp \& Paunzen 2002) seem excluded for Vega. Some form of dust - gas separation, such as suggested by Venn \& Lambert (1990) or Holweger (1992), may be involved but if so the mechanism likely must work at the time of Vega's formation since so much mass is involved.

\subsection{Determination of the Age and Mass of Vega}

We estimate the mass and age of Vega by locating its measured luminosity and polar radius in an appropriate evolution grid, as described in Peterson et al. (2006b). The interior models we adopt are from the BASTI databas 3 (Pietrinferni 2006, and references therein) which include evolutionary calculations using scaled solar and alphaenhanced compositions for stellar masses up to $2.4 M_{\odot}$. The composition found here is not a perfect fit to either of the those mixtures, but the large enhancement of oxygen is about the same compared to the heavy metals as the alphaenhanced mixture adopted there. Missing are the other alpha-rich elements at the enhanced levels, but given the dominance of oxygen even among these nuclei, that grid should give results more than adequate. To quantify how much the mismatch in the details of the distribution of abundances might affect the estimate we also calculate the mass and age using the scaled solar grid. In both cases, the heavy element fraction used is $\mathrm{Z}=0.0093_{-0.0005}^{+0.0006}$ as calculated from Table 2 and assuming $\left[N_{\mathrm{el}} / N_{\mathrm{tot}}\right]=-0.7$ for abundances not obtained here.

\footnotetext{
${ }^{3}$ www.te.astro.it/BASTI/index.php
}

For the alpha-enhanced composition we obtain $2.09 \pm 0.03 M_{\odot}$ and $536 \pm 29 \mathrm{Myr}$ for the mass and age. The simple scaled solar abundances in turn yield $2.14 M_{\odot}$ and $541 \mathrm{Myr}$ and since the alpha-enhanced models are a much closer match to Vega's composition it is clear the errors introduced by the slight mismatch are small compared to the other uncertainties.

As described in Peterson et al. (2006b), whether Vega is solar composition throughout or the derived abundances represent the actual overall composition, results in quite different estimates for the star's mass and age. Most previous authors have assumed an underlying solar composition yielding estimates of $2.3 M_{\odot}$ for the mass and an age in the neighborhood of $360 \mathrm{Myr}$. Since there is a distinct possibility that the composition we have derived applies to the star as a whole, Vega's estimated mass may be reduced and its implied age increased substantially. One immediate consequence of this is a growing clash with the properties of the so-called "Castor moving group" (Barrado y Navascués 1998), which includes Castor $(\alpha \mathrm{Gem})$, Fomalhaut ( $\alpha$ PsA) and Alderamin ( $\alpha$ Cep), in addition to Vega, and whose members are estimated to have an age of $200 \pm 100 \mathrm{Myr}$. Even with an assumed solar composition Vega's age was not a comfortable fit for inclusion in this group. The increased age we propose would make it an unlikely member.

This research was supported in part by a grant from the Naval Research Laboratory to D. M. P. and in part by NSF grant 06-07612 to Dr. Michal Simon. We also thank Dr. Robert L. Kurucz for extensive discussions.

\section{REFERENCES}

Adelman, S. J., \& Gulliver, A. F. 1990, ApJ, 348, 712

Allende Prieto, C., et al. 1999, ApJ, 527, 879

Armstrong, J. T., et al. 1998, ApJ, 496, 550

Aufdenberg, J. P., et al. 2006, ApJ, 645, 664

Barrado y Navascués, D. 1998, A\&A, 339, 831

Baschek, B., \& Slettebak, A. 1988, A\&A, 207, 112 
Baschek, B. 1992, The Atmospheres of Early-Type Stars, in Lecture Notes in Physics, ed. U. Heber and C. S. Jeffery. (Springer-Verlag, Berlin Heidelberg New York) 401, 224

Castelli, F., \& Faraggiana, R. 1979, A\&A, 79,174

Charbonneau, P. 1993, IAU Colloq. 138, Peculiar versus Normal Phenomena in A-type and Related Stars, ed. M. M. Dworetsky, F. Castelli, \& R. Faraggiana (San Francisco: ASP), 474

Castelli, F., \& Kurucz, R. L. 2003, in IAU Symp. 210, Modeling of Stellar Atmospheres, ed. N. E. Piskunov., W. W. Weiss, \& D. F. Gray (San Francisco: ASP), poster A20 on the enclosed CD-ROM (astro-ph/040587)

Ekström, S., et al. 2008, A\&A, 478, 467

Gigas, D. 1986, A\&A, 165, 170

Gray, R. O. 1988, JRASC, 82, 336

Grevesse, N., \& Sauval, A. J. 1998, Space Sci. Rev., 85, 161

Gulliver, A. F., Hill, G., \& Adelman, S. J. 1994, ApJ, 429, L81

Heiter, U. 2002, A\&A, 381, 959

Hill, G., Gulliver, A. F., \& Adelman, S. J. 2004, in IAU Symp. 224, The A-Star Puzzle, ed. J. Zverko, J. Ziznovsky, S. J. Adelman, \& W. W. Weiss (Cambridge: Cambridge Univ. Press), 35

Holweger, H. 1992, The Atmospheres of EarlyType Stars, in Lecture Notes in Physics, ed. U. Heber and C. S. Jeffery. (Springer-Verlag, Berlin Heidelberg New York) 401, 48

Holweger, H., \& Stürenburg, S. 1993, IAU Colloq. 138, Peculiar versus Normal Phenomena in A-type and Related Stars, ed. M. M. Dworetsky, F. Castelli, \& R. Faraggiana (San Francisco: ASP), 356

Ilijić, S., et al. 1998, CoSka, 27, 461

Johnson, J. A. 2002, ApJS, 139, 219

Kamp, I., \& Paunzen, E. 2002, MNRAS, 335, L45

Kurucz, R. L., \& Bell, B. 1995, CD-Rom No. 23, Smithsonian Astrophysical Observatory
Meynet, G., \& Maeder, A. 1997, A\&A, 321, 465

Monnier, J. D., et al. 2007, Science, 317, 342

Moultaka, J., et al. 2004, PASP, 116, 693

Paunzen, E., et al. 1999, A\&A, 345, 597

Peterson, D. M., et al 2004, SPIE, 5491, 65

Peterson, D. M., et al. 2006a, ApJ, 636, 1087

Peterson, D. M., et al. 2006b, Nature, 440, 896

Petrie, R. M. 1964, Publ. Dom. Astrophys. Obs. Victoria, 12, 317

Pietrinferni, A., et al. 2006, AJ, 642, 797

Pradhan, A., et al. 1995, BAAS, 27, 841

Sadakane, K., \& Nishimura, M. 1981, PASJ, 33, 189

Takeda, Y., Kawanomoto, S., \& Ohishi, N. 2007, PASJ, 59, 245

Takeda, Y., Kawanomoto, S., \& Ohishi, N. 2008, ApJ, preprint doi:10.1086/'528949'

Talon, S., et al. 1997, A\&A, 322, 209

ten Brummelaar, T. A., et al. 2005, ApJ, 628, 453

Thévenin, F., \& Idiart, T. P. 1999, ApJ, 521, 753

Venn, K. A., \& Lambert, D. L. 1993, ApJ, 363, 234

von Zeipel, H. 1924, MNRAS, 84, 684

Zahn, J. -P. 1992, A\&A, 256, 115

This 2-column preprint was prepared with the AAS LATEX macros v5.2. 
TABLE 1

The Abundance Analysis of Vega

\begin{tabular}{|c|c|c|c|c|c|}
\hline $\begin{array}{c}\lambda \\
(\AA)\end{array}$ & $\begin{array}{c}\mathrm{EP} \\
\left(\mathrm{cm}^{-1}\right)\end{array}$ & $\begin{array}{c}w_{\lambda} \\
(\mathrm{m} \AA)\end{array}$ & $\log g f$ & $\log \frac{N_{\mathrm{el}} \mathrm{a}}{N_{\mathrm{tot}}}$ & Blends \\
\hline \multicolumn{6}{|c|}{ He I $\left(\frac{N_{\mathrm{He}}}{N_{\mathrm{tet}}}=0.072 \pm 0.004\right)$} \\
\hline 4471.498 & 169087.008 & $\ldots$ & 0.052 & 0.070 & \\
\hline 4713.139 & 169086.864 & 5 & -1.233 & 0.078 & Fe II $\lambda 4713.193$ \\
\hline 4921.931 & 171135.000 & 8 & -0.435 & 0.060 & \\
\hline 5015.678 & 166277.546 & $\cdots$ & -0.820 & 0.078 & Fe II $\lambda 5015.755$ \\
\hline 6678.154 & 171135.000 & 5 & 0.329 & 0.070 & Fe II $\lambda 6677.306$ \\
\hline \multicolumn{6}{|c|}{$\mathrm{C}_{\text {I }}\left(\log \frac{N_{\mathrm{C}}}{N_{\mathrm{t}} \mathrm{t}}=-4.14 \pm 0.04,\left[N_{\mathrm{C}} / N_{\text {tot }}\right]^{\mathrm{b}}=-0.62\right)$} \\
\hline 4770.021 & ot 60352.639 & 7 & -2.052 & -4.16 & \\
\hline 4771.730 & 60393.148 & 25 & -1.488 & -4.16 & \\
\hline 4775.889 & 60393.148 & 7 & -2.013 & -4.16 & \\
\hline 4932.050 & 61981.818 & 16 & -1.574 & -4.06 & \\
\hline \multicolumn{6}{|c|}{ O I $\left(\log \frac{N_{\mathrm{O}}}{N_{\mathrm{tat}}}=-3.32 \pm 0.04,\left[N_{\mathrm{O}} / N_{\text {tot }}\right]=-0.11\right)$} \\
\hline 5329.099 & ot 86625.757 & $34^{\mathrm{c}}$ & -1.730 & -3.31 & \\
\hline 5329.690 & 86627.778 & $\cdots$ & -1.410 & -3.31 & \\
\hline 5330.741 & 86631.454 & 24 & -1.120 & -3.31 & \\
\hline 6046.438 & 88631.146 & 10 & -1.675 & -3.26 & \\
\hline 6155.971 & 86625.757 & $77^{\mathrm{d}}$ & -1.051 & -3.36 & \\
\hline 6156.778 & 86627.778 & $\cdots$ & -0.731 & -3.36 & \\
\hline 6158.187 & 86631.454 & 59 & -0.441 & -3.36 & \\
\hline \multicolumn{6}{|c|}{$\mathrm{Mg}$ I $\left(\log \frac{N_{\mathrm{Mg}}}{N_{\text {tot }}}=-5.12 \pm 0.05,\left[N_{\mathrm{Mg}} / N_{\text {tot }}\right]=-0.66\right)$} \\
\hline 4702.991 & tot 35051.264 & 29 & -0.666 & -5.06 & \\
\hline 5167.321 & 21850.405 & 81 & -1.030 & -5.06 & Fe I $\lambda 5167.488$ \\
\hline 5172.684 & 21870.464 & 102 & -0.402 & -5.16 & \\
\hline 5183.604 & 21911.178 & 119 & -0.180 & -5.16 & \\
\hline 5528.405 & 35051.264 & 28 & -0.620 & -5.16 & \\
\hline \multicolumn{6}{|c|}{$\mathrm{Mg}$ II $\left(\log \frac{N_{\mathrm{Mg}}}{N_{\mathrm{tot}}}=-5.06 \pm 0.04,\left[N_{\mathrm{Mg}} / N_{\mathrm{tot}}\right]=-0.6\right)$} \\
\hline 4427.994 & $\begin{array}{l}\text { tot } \\
80619.500\end{array}$ & $\ldots$ & -1.210 & -5.06 & \\
\hline 4433.988 & 80650.020 & $\ldots$ & -0.910 & -5.11 & Fe I $\lambda 4433.782$ \\
\hline 4481.126 & 71490.190 & $\cdots$ & 0.740 & -5.01 & \\
\hline \multicolumn{6}{|c|}{$\mathrm{Al} \mathrm{II}\left(\log \frac{N_{\mathrm{Al}}}{N_{\text {tot }}}=-6.22,\left[N_{\mathrm{Al}} / N_{\text {tot }}\right]=-0.65\right)$} \\
\hline 4663.046 & 85481.350 & $\ldots$ & -0.284 & -6.22 & \\
\hline \multicolumn{6}{|c|}{ Si II $\left(\log \frac{N_{\mathrm{Si}}}{N_{\text {tot }}}=-5.15 \pm 0.05,\left[N_{\mathrm{Si}} / N_{\text {tot }}\right]=-0.66\right)$} \\
\hline 4128.054 & ${ }^{\text {tot }} 79338.500$ & 32 & 0.316 & -5.19 & Mn II $\lambda 4128.129$ \\
\hline 4130.872 & 79355.020 & 54 & -0.824 & -5.19 & \\
\hline 5055.984 & 81251.320 & 60 & 0.593 & -5.19 & \\
\hline 6347.109 & 65500.470 & 118 & 0.297 & -5.09 & $\begin{array}{l}\text { Mg iा } \lambda 6346.742 \\
M g \text { II } \lambda 6346.964\end{array}$ \\
\hline 6371.371 & 65500.470 & 82 & -0.003 & -5.09 & \\
\hline \multicolumn{6}{|c|}{ S I $\left(\log \frac{N_{\mathrm{S}}}{N_{\mathrm{tot}}}=-5.01,\left[N_{\mathrm{S}} / N_{\text {tot }}\right]=-0.3\right)$} \\
\hline 6052.674 & 63475.051 & 7 & -0.740 & -5.01 & \\
\hline \multicolumn{6}{|c|}{ Ca I $\left(\log \frac{N_{\mathrm{Ca}}}{N_{\mathrm{tat}}}=-6.72 \pm 0.12,\left[N_{\mathrm{Ca}} / N_{\mathrm{tot}}\right]=-1.04\right)$} \\
\hline 4226.728 & tot $\quad 0.000$ & $\ldots$ & 0.243 & -6.73 & \\
\hline 4434.957 & 15210.063 & $\ldots$ & -0.029 & -6.73 & \\
\hline 4585.865 & 20371.000 & 1 & -0.386 & -6.68 & \\
\hline 5588.749 & 20371.000 & 1 & 0.210 & -6.63 & \\
\hline 5594.462 & 20349.260 & 5 & -0.050 & -6.63 & \\
\hline 5598.480 & 20335.360 & 4 & -0.220 & -6.63 & Fe I $\lambda 5598.287$ \\
\hline 6162.173 & 15315.943 & 9 & 0.100 & -6.98 & \\
\hline
\end{tabular}


TABle 1-Continued

\begin{tabular}{|c|c|c|c|c|c|}
\hline $\begin{array}{c}\lambda \\
(\AA)\end{array}$ & $\begin{array}{c}\mathrm{EP} \\
\left(\mathrm{cm}^{-1}\right)\end{array}$ & $\begin{array}{c}w_{\lambda} \\
(\mathrm{m} \AA)\end{array}$ & $\log g f$ & $\log {\frac{N_{\mathrm{el}}}{N_{\mathrm{tot}}}}^{\mathrm{a}}$ & Blends \\
\hline 4246.822 & 2540.950 & 5 & 0.320 & -10.02 & \\
\hline 5526.79 & 14261.320 & 9 & 0.130 & -9.92 & \\
\hline \multicolumn{6}{|c|}{ Ti II $\left(\log \frac{N_{\mathrm{Ti}}}{N_{\mathrm{tot}}}=-7.65 \pm 0.09,\left[N_{\mathrm{Ti}} / N_{\mathrm{tot}}\right]=-0.63\right)$} \\
\hline 4468.507 & $\begin{array}{l}\text { tot } \\
\quad 9118.260\end{array}$ & 70 & -0.600 & -7.82 & \\
\hline 4529.474 & 12676.970 & 9 & -1.830 & -7.69 & \\
\hline 4563.761 & 9850.900 & 57 & -1.010 & -7.51 & \\
\hline 4589.958 & 9975.920 & 16 & -1.790 & -7.61 & Cr II $\lambda 4589.901$ \\
\hline 4708.665 & 9975.920 & 3 & -2.410 & -7.69 & \\
\hline 4779.985 & 16515.860 & 12 & -1.420 & -7.59 & \\
\hline 4805.085 & 16625.110 & 21 & -1.100 & -7.59 & \\
\hline 5336.771 & 12758.110 & 12 & -1.700 & -7.72 & \\
\hline \multicolumn{6}{|c|}{ Cr II $\left(\log \frac{N_{\mathrm{Cr}}}{N_{\mathrm{tot}}}=-6.91 \pm 0.1,\left[N_{\mathrm{Cr}} / N_{\text {tot }}\right]=-0.54\right)$} \\
\hline 4252.632 & 31117.390 & 6 & -2.018 & -6.97 & \\
\hline 4261.847 & 25033.700 & 18 & -3.004 & -6.92 & Cr II $\lambda 4261.913$ \\
\hline 4554.988 & 32836.680 & 20 & -1.430 & -6.87 & \\
\hline 4558.650 & 32854.310 & 61 & -0.660 & -6.87 & \\
\hline 4565.740 & 32603.400 & 7 & -1.910 & -7.07 & \\
\hline 4588.199 & 32836.680 & 48 & -0.830 & -6.87 & \\
\hline 4592.049 & 32854.950 & 18 & -1.420 & -6.87 & \\
\hline 4616.629 & 32844.760 & 16 & -1.530 & -6.87 & \\
\hline 4618.803 & 32854.950 & 36 & -1.070 & -6.87 & \\
\hline 4634.070 & 32844.760 & 29 & -1.220 & -6.82 & \\
\hline 4812.337 & 31168.580 & 6 & -1.930 & -7.07 & \\
\hline 4824.127 & 31219.350 & 39 & -1.220 & -6.72 & \\
\hline 5334.869 & 32844.760 & 10 & -1.562 & -7.07 & \\
\hline \multicolumn{6}{|c|}{ Mn I $\left(\log \frac{N_{\mathrm{Mn}}}{N_{\text {tot }}}=-7.45,\left[N_{\mathrm{Mn}} / N_{\text {tot }}\right]=-0.8\right)$} \\
\hline 4783.405 & 18531.663 & 2 & 0.042 & -7.45 & \\
\hline \multicolumn{6}{|c|}{ Fe I $\left(\log \frac{N_{\mathrm{Fe}}}{N_{\mathrm{tot}}}=-5.51 \pm 0.1,\left[N_{\mathrm{Fe}} / N_{\mathrm{tot}}\right]=-0.97\right)$} \\
\hline 4132.058 & 12968.553 & 29 & -0.650 & -5.54 & $\begin{array}{l}\text { Fe г } \lambda 4131.935 \\
\text { Fe г } \lambda 4131.971\end{array}$ \\
\hline 4134.677 & 22838.321 & 8 & -0.490 & -5.54 & Fe I $\lambda 4134.42$ \\
\hline 4136.998 & 27543.001 & 4 & -0.540 & -5.54 & \\
\hline 4250.119 & 19912.494 & 18 & -0.405 & -5.49 & Fe II $\lambda 4250.437$ \\
\hline 4250.787 & 12560.933 & 27 & -0.710 & -5.49 & Fe II $\lambda 4250.437$ \\
\hline 4260.474 & 19350.890 & 36 & -0.020 & -5.39 & \\
\hline 4466.551 & 22838.321 & 9 & -0.590 & -5.36 & \\
\hline 4476.019 & 22946.814 & 8 & -0.570 & -5.79 & Fe I $\lambda 4476.076$ \\
\hline 4528.614 & 17550.180 & 15 & -1.072 & -5.51 & \\
\hline 4918.994 & 23110.937 & 16 & -0.640 & -5.51 & Fe I $\lambda 4918.954$ \\
\hline 4920.502 & 22845.867 & 27 & -3.955 & -5.51 & Cr iा $\lambda 4920.23$ \\
\hline 5324.179 & 25899.987 & 12 & -0.240 & -5.49 & \\
\hline 5586.756 & 27166.818 & 11 & -0.210 & -5.59 & Fe II $\lambda 5587.114$ \\
\hline 5615.644 & 26874.548 & 15 & -0.140 & -5.44 & \\
\hline \multicolumn{6}{|c|}{ Fe II $\left(\log \frac{N_{\mathrm{Fe}}}{N_{\mathrm{tat}}}=-5.12 \pm 0.09,\left[N_{\mathrm{Fe}} / N_{\mathrm{tot}}\right]=-0.58\right)$} \\
\hline 4258.154 & $\begin{aligned} \text { tot } \\
21812.055\end{aligned}$ & 14 & -0.467 & -5.34 & Fe II $\lambda 4258.34$ \\
\hline 4520.224 & 22637.205 & 45 & -2.990 & -5.13 & \\
\hline 4522.634 & 22939.358 & 69 & -2.700 & -5.01 & \\
\hline 4576.340 & 22939.358 & 23 & -3.390 & -5.06 & \\
\hline 4582.835 & 22939.358 & 17 & -3.570 & -5.06 & \\
\hline 4583.837 & 22637.205 & 88 & -2.490 & -4.97 & Fe iा $\lambda 4583.999$ \\
\hline 4596.015 & 50212.826 & $\ldots$ & -2.057 & -5.21 & Fe Iі $\lambda 4595.682$ \\
\hline 4620.521 & 22810.357 & 14 & -3.650 & -5.16 & \\
\hline 4635.316 & 48039.090 & 13 & -1.650 & -5.21 & \\
\hline
\end{tabular}


TABLE 1-Continued

\begin{tabular}{|c|c|c|c|c|c|}
\hline $\begin{array}{c}\lambda \\
(\AA)\end{array}$ & $\begin{array}{c}\mathrm{EP} \\
\left(\mathrm{cm}^{-1}\right)\end{array}$ & $\begin{array}{c}w_{\lambda} \\
(\mathrm{m} \AA)\end{array}$ & $\log g f$ & $\log {\frac{N_{\mathrm{el}}}{N_{\mathrm{tot}}}}^{\mathrm{a}}$ & Blends \\
\hline 4656.981 & 23317.633 & 12 & -3.950 & -5.11 & Ti iा $\lambda 4657.206$ \\
\hline 4663.708 & 23317.633 & 5 & -4.145 & -5.11 & \\
\hline 4666.758 & 22810.357 & 11 & -3.700 & -5.01 & \\
\hline 4670.182 & 20830.582 & 8 & -4.350 & -5.11 & Sc II $\lambda 4670.407$ \\
\hline 4923.927 & 23317.633 & 114 & -1.820 & -5.06 & \\
\hline 5534.847 & 26170.181 & 25 & -2.930 & -5.09 & \\
\hline 6147.741 & 31364.440 & 14 & -2.721 & -5.24 & \\
\hline 6149.258 & 31368.450 & 13 & -2.724 & -5.24 & \\
\hline \multicolumn{6}{|c|}{ Ni I $\left(\log \frac{N_{\mathrm{Ni}}}{N_{\text {tot }}}=-6.79,\left[N_{\mathrm{Ni}} / N_{\text {tot }}\right]=-1.0\right)$} \\
\hline 4714.417 & 27260.894 & 3 & 0.160 & -6.79 & \\
\hline \multicolumn{6}{|c|}{ Ba II $\left(\log \frac{N_{\mathrm{Ba}}}{N_{\text {tot }}}=-11.21,\left[N_{\mathrm{Ba}} / N_{\text {tot }}\right]=-1.3\right)$} \\
\hline 4554.029 & 0.000 & 13 & 0.430 & -11.21 & \\
\hline 4934.076 & 0.000 & 7 & -0.150 & -11.21 & Fe I $\lambda 4934.005$ \\
\hline
\end{tabular}

${ }^{\mathrm{a}}$ For helium abundance, $\frac{N_{\mathrm{He}}}{N_{\text {tot }}}$

${ }^{\mathrm{b}}\left[N_{\mathrm{el}} / N_{\text {tot }}\right]=\log \frac{N_{\mathrm{el}}}{N_{\text {tot }}}-\log \left(\frac{N_{\mathrm{el}}}{N_{\text {tot }}}\right) \odot$

${ }^{\mathrm{c}}$ The equivalent width is for the blend with O I $\lambda 5629.690$.

${ }^{d}$ The equivalent width is for the blend with $\mathrm{O}_{\text {I }} \lambda 6156.778$. 
Table 2: Comparisons with the previous abundance studies

\begin{tabular}{|c|c|c|c|c|}
\hline \multirow{2}{*}{$\begin{array}{l}\text { Atomic } \\
\text { Species }\end{array}$} & \multicolumn{2}{|c|}{$\log \mathrm{M} / \mathrm{H}$} & $\log \frac{N_{\text {el }} \mathrm{c}}{N_{\text {to }}}$ & {$\left[N_{\mathrm{el}} / N_{\text {tot }}\right]^{\mathrm{d}}$} \\
\hline & $\mathrm{SN}^{\mathrm{a}}$ & $\mathrm{AG}^{\mathrm{b}}$ & \multicolumn{2}{|c|}{ This work } \\
\hline$\overline{\mathrm{He} \mathrm{I}}$ & $\cdots$ & -1.52 & -1.14 & -0.04 \\
\hline C I & $\cdots$ & -3.81 & -4.14 & -0.62 \\
\hline $\mathrm{NI}^{\mathrm{e}}$ & $\cdots$ & $\ldots$ & -4.53 & +0.09 \\
\hline O I & $\cdots$ & $\cdots$ & -3.32 & -0.11 \\
\hline Mg I & -4.61 & -5.07 & -5.12 & -0.66 \\
\hline Mg II & -4.96 & -5.11 & -5.06 & -0.60 \\
\hline $\mathrm{Al}$ II & $\cdots$ & -6.33 & -6.22 & -0.65 \\
\hline Si II & .. & $\cdots$ & -5.15 & -0.66 \\
\hline S I & $\cdots$ & $\cdots$ & -5.01 & -0.30 \\
\hline $\mathrm{Ca} \mathrm{I}$ & -6.11 & -6.21 & -6.72 & -1.04 \\
\hline Sc II & -9.42 & -9.62 & -9.97 & -1.10 \\
\hline Ti II & -7.31 & -7.47 & -7.65 & -0.63 \\
\hline Cr II & -6.90 & -6.76 & -6.91 & -0.54 \\
\hline Mn I & -6.87 & -7.16 & -7.45 & -0.80 \\
\hline Fe I & -5.09 & -5.05 & -5.51 & -0.97 \\
\hline Fe II & -5.09 & -5.12 & -5.12 & -0.61 \\
\hline Ni I & -5.94 & -6.38 & -6.79 & -1.00 \\
\hline Ba II & -10.25 & -10.58 & -11.21 & -1.30 \\
\hline
\end{tabular}

Note. - The definition of the abundances we use differs from that adopted by SN and AG. For the helium abundance found here, the SN and AG abundances will be systematically larger than ours by 0.03 dex.

$a$ Sadakane \& Nishimura (1981)

Adelman \& Gulliver (1990)

${ }^{c}$ Abundances from Table 1

${ }^{d}$ Solar abundances have been taken from Grevesse \& Sauval 1998)

${ }^{e}$ Abundance based on Venn \& Lambert (1990) equivalent widths 